\title{
Effect of CdSe quantum dots on hole transport in poly(3-hexylthiophene) thin films
}

\author{
Kusum Kumari, ${ }^{1, a)}$ Suresh Chand, ${ }^{2}$ Pankaj Kumar, ${ }^{2}$ Shailesh N. Sharma, ${ }^{2}$ V. D. Vankar, ${ }^{1}$ \\ and Vikram Kumar ${ }^{2}$ \\ ${ }^{1}$ Nanoscience and Nanotechnology Laboratory, Department of Physics, Indian Institute of Technology Delhi, \\ New Delhi 110 016, India \\ ${ }^{2}$ Materials Division, National Physical Laboratory, Dr. K. S. Krishnan Road, New Delhi 110 012, India
}

(Received 11 April 2008; accepted 6 June 2008; published online 3 July 2008)

\begin{abstract}
This letter demonstrates the effect of cadmium selenide $(\mathrm{CdSe})$ quantum dots on hole transport in poly(3-hexylthiophene) (P3HT) thin films. Current-voltage characteristics of P3HT and P3HT:CdSe thin films have been studied in the temperature range of $288-85 \mathrm{~K}$, in hole only device configurations, i.e., indium tin oxide (ITO)/poly(ethylene-dioxthiophene):polystyrenesulphonate (PEDOT:PSS)/P3HT/Au and ITO/PEDOT:PSS/P3HT:CdSe/Au. The incorporation of CdSe quantum dots in $\mathrm{P} 3 \mathrm{HT}$ results in the enhancement in hole current and switches the transport from dual conduction mechanism, viz., trap and mobility models to only trap model. This is attributed to the reduction in characteristic trap energy from 60 to $32 \mathrm{meV}$ and trap density from 2.5 $\times 10^{18}$ to $1.7 \times 10^{18} \mathrm{~cm}^{-3}$. (C) 2008 American Institute of Physics. [DOI: 10.1063/1.2955524]
\end{abstract}

Polymer-fullerene interpenetrating bulk heterojunctions (BHJs) are currently the most efficient approach in organic photovoltaics. Power conversion efficiency $\sim 6 \%$ has already been realized ${ }^{1}$ in poly (3-hexylthiophene): [6,6]-phenyl-C61 butyric acid methyl ester (P3HT:PCBM) BHJs. However, there is a strong need to search for innovative ways to enhance further the efficiency and stability of these polymer solar cells. One of the promising approaches is the incorporation of different ${ }^{2-5}$ inorganic nanoparticles such as titanium dioxide $\left(\mathrm{TiO}_{2}\right)$, cadmium telluride $(\mathrm{CdTe})$, cadmium selenide telluride ( $\mathrm{CdSeTe})$, zinc oxide $(\mathrm{ZnO})$, etc., into host conducting polymers such as poly[2-methoxy, 4-(2-ethylhexoxy)1,3-phenylene vinylene] (MEH-PPV) and P3HT. Encouraging results are already available in literature on the preparation of solar cells using polymer $\mathrm{P} 3 \mathrm{HT}$ and $\mathrm{CdSe}$ nanostructures. ${ }^{6-8}$ To improve further the performance of P3HT:CdSe based polymer solar cells it is essential to understand their device physics especially the role of nanoparticles/quantum dots on charge transport properties of the composite matrix. In this letter we have investigated the effect of dispersion of CdSe quantum dots on hole transport in P3HT thin films. Current-voltage $(J-V)$ characteristics of P3HT thin films were studied in hole only device configuration, i.e., indium tin oxide (ITO)/poly(ethylene-dioxythiophene):polystyrenesulphonate (PEDOT:PSS)/P3HT/Au in the temperature range of $288-85 \mathrm{~K}$, without and with dispersion of CdSe quantum dots of size $\sim 5 \mathrm{~nm}$. It has been found here that the incorporation of CdSe quantum dots in P3HT switches its hole transport from a dual conduction mechanism, viz., trap and mobility models to only trap model. This finding explicitly reveals the pivotal role played by $\mathrm{CdSe}$ quantum dots in governing charge transport of P3HT:CdSe composite films that would prove beneficial in the development of improved solar cells based on organicinorganic composites.

Samples for both pure P3HT and P3HT:CdSe composite films were prepared under identical conditions in hole only

\footnotetext{
${ }^{\text {a) }}$ Author to whom correspondence should be addressed. Electronic mail:
} kusumiitd@gmail.com. device configurations, i.e., ITO $(\varphi \sim 4.8 \mathrm{eV}) /$ PEDOT:PSS $(80 \mathrm{~nm}) / \mathrm{P} 3 \mathrm{HT}(\sim 180 \mathrm{~nm}) / \mathrm{Au} \quad(\varphi \sim 5.2 \mathrm{eV})$ and ITO $(\varphi$ $\sim 4.8 \mathrm{eV}) / \mathrm{PEDOT}:$ PSS $(80 \mathrm{~nm}) / \mathrm{P} 3 \mathrm{HT}: \mathrm{CdSe}(\sim 180 \mathrm{~nm}) / \mathrm{Au}$ $(\varphi \sim 5.2 \mathrm{eV})$ and were designated as devices 1 and 2 , respectively. Thin film of PEDOT:PSS (Aldrich USA) was spin coated onto precleaned and plasma treated ITO (sheet resistance $\sim 18 \Omega / \square$ ) coated glass substrates at $2000 \mathrm{rpm}$ and cured at $120^{\circ} \mathrm{C}$ for $30 \mathrm{~min}$ in vacuum. Regioregular P3HT obtained from Aldrich USA was used as such without any further purification. CdSe quantum dots $(\sim 5 \mathrm{~nm})$ were synthesized by well known wet chemical route ${ }^{9}$ using cadmium oxide, selenium powder as precursor, and trioctyl phosphene oxide (TOPO) as capping agent. To achieve better charge transport properties between the CdSe quantum dots and the host polymer P3HT capping agent TOPO was removed by treatment with pyridine. For this, the passivated CdSe particles were washed several times in methanol, dissolved in pyridine, and precipitated with hexanes. ${ }^{6,8}$ Subsequently, two homogeneous solutions were made, i.e., one by dissolving P3HT in chloroform with material content of $10 \mathrm{mg} / \mathrm{ml}$ and other by dispersing CdSe quantum dots in P3HT (1:1 weight ratio) in binary solvent of chloroform:pyridine (9:1 volume ratio). Figure 1 shows the transmission electron microscopy (TEM) image of CdSe quantum dots dispersed in host P3HT in ratio $1: 1$ by weight. The TEM image quite explicitly reveals the monodisperse and uniform distribution of $\mathrm{CdSe}$ quantum dots in P3HT matrix with an average particle size $\sim 5 \mathrm{~nm}$. P3HT and P3HT:CdSe composite thin films were spin cast in an inert atmosphere on the PEDOT:PSS films at $2000 \mathrm{rpm}$, and cured at $120{ }^{\circ} \mathrm{C}$ for $15 \mathrm{~min}$. On top of these films, Au electrodes $\sim 400 \mathrm{~nm}$ were deposited by vacuum thermal evaporation at $5 \times 10^{-6}$ Torr.

The devices so obtained were sealed using ultraviolet irradiated epoxy resin. $J-V$ characteristics of these samples in the temperature range of $288-85 \mathrm{~K}$ were measured using a low temperature setup coupled with a Keithley 2400 Source Meter unit interfaced with a PC.

Relevant theoretical details used for the analysis of experimental results are given here. Earlier studies in organics have shown that the space charge limited conduction 


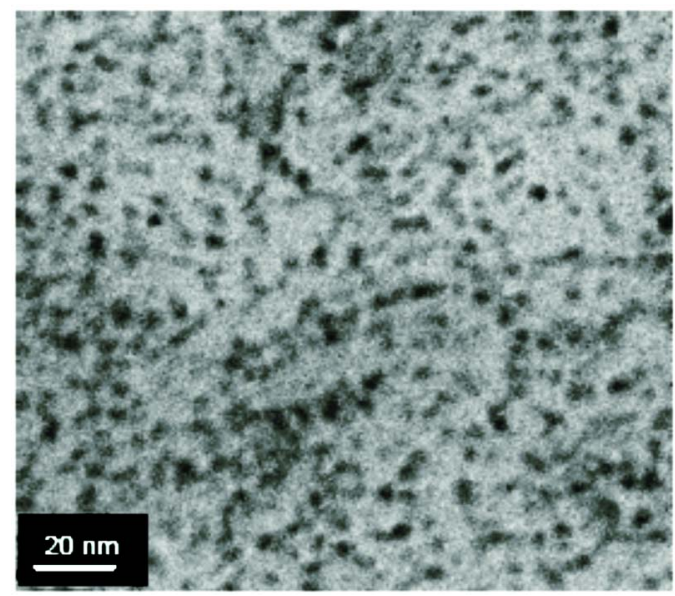

FIG. 1. (Color online) TEM image of CdSe quantum dots dispersed in P3HT matrix in 1:1 weight ratio.

governs ${ }^{10,11}$ the charge transport either ${ }^{12,13}$ by drift of charge carriers under the influence of traps, distributed in energy space, called trap model or by temperature and field dependent mobility models. The former mechanism dominates at higher temperature and the latter at lower temperatures $(<200 \mathrm{~K})$.

Assuming that the trapped carrier density $\left(p_{t}\right) \gg$ free carrier density $(p)$ and using continuity equation and boundary condition for current density $(J)$ and applied voltage $(V)$ as

$$
\begin{aligned}
& J=q \mu p(x) F(x), \\
& V=\int F(x) d x,
\end{aligned}
$$

the expression for $J$, for traps $\left(N_{t}\right)$ distributed exponentially in energy $(E)$ as

$$
N_{t}(E)=\left(H_{b} / E_{t}\right) \exp \left(-E / E_{t}\right),
$$

is given by ${ }^{14}$

$$
J=q^{1-l} \mu N_{v}\left(\frac{2 l+1}{l+1}\right)^{l+1}\left(\frac{l}{l+1} \frac{\varepsilon \varepsilon_{0}}{H_{b}}\right)^{l} \frac{V^{l+1}}{d^{2 l+1}},
$$

where $q$ is the elementary charge, $\mu$ is the charge carrier mobility, $N_{\nu}$ is the effective density of states, $d$ is the film thickness, $F$ is the applied electric field, $\varepsilon$ is the dielectric constant of material, $\varepsilon_{0}$ is the permittivity of the free space, $H_{b}$ is the density of traps, $E_{t}$ is the characteristic trap energy which is often expressed in terms of the characteristics temperature of trap distribution $T_{c}$ as $E_{t}=k_{B} T_{c}$, and $l=E_{t} / k_{B} T$ $=T_{c} / T$ where $k_{B}$ is Boltzmann's constant.

The mobility model suggests that the mobility in these materials obey the empirical law ${ }^{12}$

$$
\mu(F, T)=\mu_{0} \exp \left[-\frac{\Delta_{0}}{k_{B} T}+\beta\left(\frac{1}{k_{B} T}-\frac{1}{k_{B} T_{0}}\right) \sqrt{F}\right],
$$

where $\Delta_{0}$ is the zero field activation energy, $\mu_{0}$ is a constant, $\beta$ is the Poole-Frenkel-like coefficient, $T_{0}$ is the temperature shift factor ranging from 400 to $600 \mathrm{~K}, T$ is the absolute temperature, and $F$ is the applied electric field. $J$ - $V$ characteristics were obtained by solving numerically the Poisson and continuity equations ${ }^{10}$ and using the value of mobility $\mu$ from Eq. (5).

Figure 2 shows the $J-V$ characteristics of device 1 at different temperatures in the range of $288-100 \mathrm{~K}$ which has been theoretically analyzed both for the trap as well as the Downloaded 29 Apr 2009 to 202.141.140.34. Redistribution subject

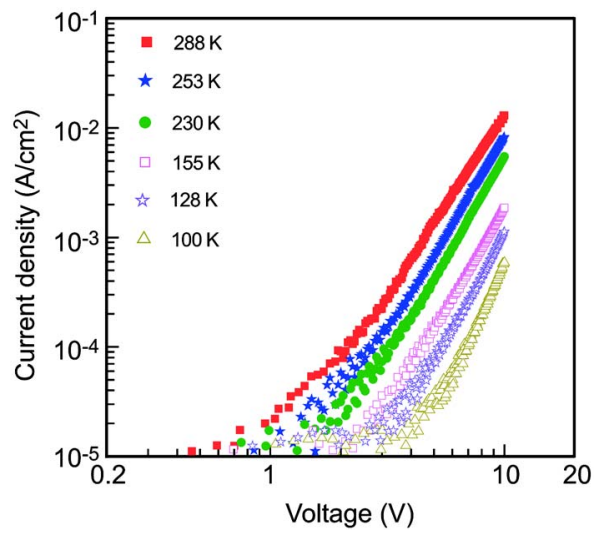

FIG. 2. (Color online) $J-V$ characteristics of device 1: ITO/PEDOT:PSS/ $\mathrm{P} 3 \mathrm{HT} / \mathrm{Au}$ in the temperature range of $288-100 \mathrm{~K}$.

mobility model using Eqs. (4) and (5), respectively. It has been found that trap model and mobility model govern the $J-V$ behavior in different temperature ranges. The exact comparison of the experimental data with the theoretically generated curves for temperature ranges of $288-230 \mathrm{~K}$ and $155-100 \mathrm{~K}$ is shown explicitly in Figs. 3(a) and 3(b), respectively. Here symbols represent the experimental data while solid and dashed lines correspond to the theoretically generated curves at corresponding temperatures using Eqs. (4) and (5), respectively. As shown by solid lines in Fig. 3(a), the experimental data in temperature range from 288 to $230 \mathrm{~K}$ agrees well with the trap model rather than the mobility model with fitting parameters as $H_{b}=2.5$ $\times 10^{18} \mathrm{~cm}^{-3}, \quad N_{\nu}=1 \times 10^{19} \mathrm{~cm}^{-3}, \quad \varepsilon=3, \quad \varepsilon_{0}=8.85$ $\times 10^{-14} \mathrm{~F} / \mathrm{cm}, \mu \sim 5.6 \times 10^{-5} \mathrm{~cm}^{2} \mathrm{~V}^{-1} \mathrm{~s}^{-1}, d=180 \mathrm{~nm}$, and $T_{c}=700 \mathrm{~K}$, which corresponds to $E_{t} \sim 60 \mathrm{meV}$. Similarly, dashed lines in Fig. 3(b) show that the experimental data
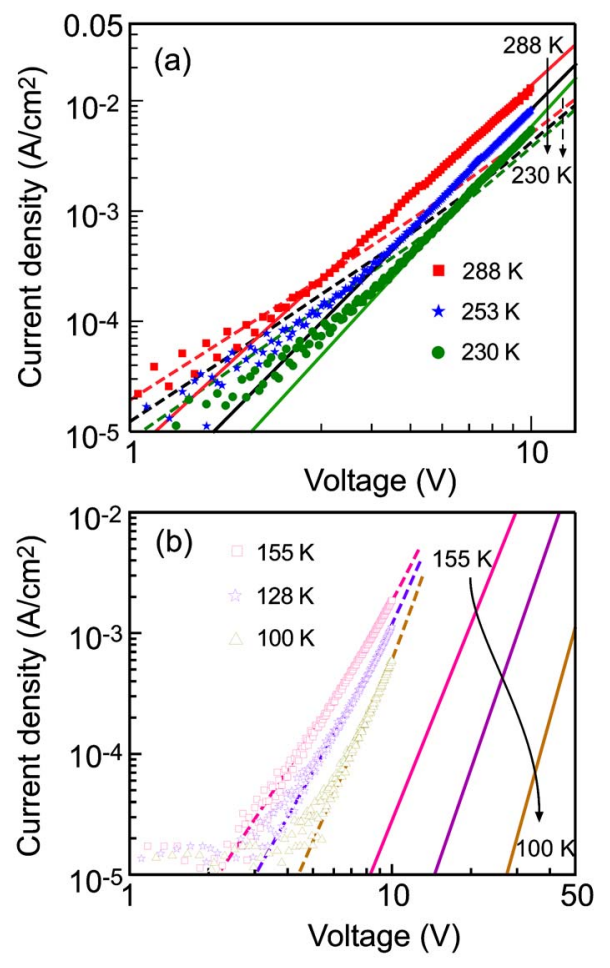

FIG. 3. (Color online) Comparison of experimental data (symbols) and theoretically generated curves [solid using Eq. (4) and dashed using Eq. (5)] at respective temperatures. (a) for temperature range of $288-230 \mathrm{~K}$ and (b) for temperature range of $155-100 \mathrm{~K}$. The values of fitting parameters for

both trap and mobility models are mentioned in the text.

o AIP license or copyright; see http://apl.aip.org/apl/copyright.jsp 


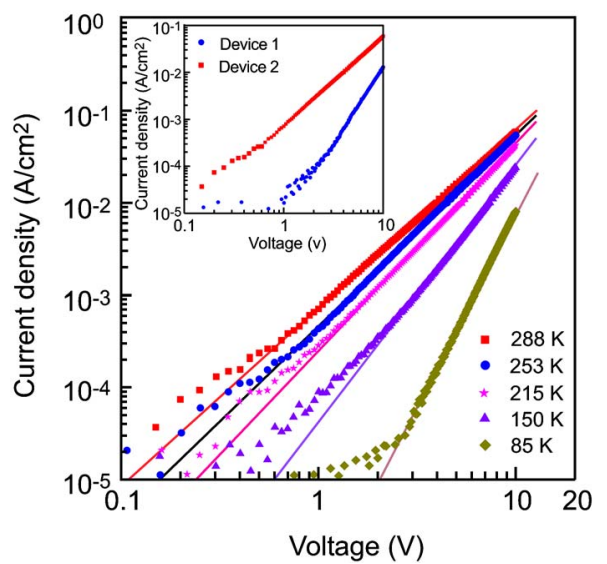

FIG. 4. (Color online) Experimental (symbols) and calculated [solid lines using Eq. (4)] $J-V$ characteristics of device 2: ITO/PEDOT: PSS/P3HT:CdSe/Au in the temperature range of $288-85 \mathrm{~K}$. The values of fitting parameters for trap model are mentioned in the text. The inset shows the comparison of $J-V$ characteristics of devices 1 and 2 at $288 \mathrm{~K}$.

from 155 to $100 \mathrm{~K}$ temperature agrees well with the mobility model rather than the trap model with fitting parameters as $\mu_{0}=1.2 \times 10^{-5} \mathrm{~cm}^{2} \mathrm{~V}^{-1} \mathrm{~s}^{-1}, \beta=1.1 \times 10^{-4} \mathrm{eV} / \mathrm{V}^{1 / 2} \mathrm{~cm}^{1 / 2}$, and $T_{0}=600 \mathrm{~K}$. Further for mobility model the activation energies at low temperatures $(155-100 \mathrm{~K})$ and different voltages were calculated from the slope of straight lines plots of $\log J$ versus $1000 / T$ (plots not shown). These values were used in calculating $\Delta_{0}$ which was found to be $0.1 \mathrm{eV}$. The $\Delta_{0}$ was obtained by the extrapolation of activation energy versus $\sqrt{F}$ plot to zero field (plot not shown). Similar discrepancy of experimental data at high and low temperatures fitting different mechanisms of conduction was also reported by Kapoor et al. ${ }^{10}$ in MEH-PPV. In fact both the mechanisms of transport, viz., trap and mobility models occur at all temperatures, however, it is only their relative magnitude that changes in going from high temperatures to low temperatures to show their dominance. At room temperature when sufficient numbers of holes are ionized from the traps to the extended states in highest occupied molecular orbital (HOMO) of the polymer then trap model becomes predominant. However, at lower temperatures, the charge carriers from traps do not posses adequate energy to go into the extended state thus they move from one localized states to another by the hopping process where mobility is field and temperature dependent and the mobility model becomes important and predominant.

Figure 4 shows the $J-V$ characteristics of device 2 at different temperatures in the range of $288-85 \mathrm{~K}$. These experimental data were analyzed for trap and mobility models using Eqs. (4) and (5), respectively. However, in this case it is interesting to note that the entire data fit well with the trap model for all temperatures. This clearly demonstrates that the hole conduction mechanism in P3HT films switched from a dual conduction mechanism, viz., trap and mobility to only trap model conduction on dispersion of $\mathrm{CdSe}$ quantum dots. The values of fitting parameters for this case were $H_{b}=1.7 \times 10^{18} \mathrm{~cm}^{-3}, \quad N_{\nu}=1 \times 10^{19} \mathrm{~cm}^{-3}, \quad \varepsilon=3, \quad \varepsilon_{0}=8.85$ $\times 10^{-14} \mathrm{~F} / \mathrm{cm}, \mu \sim 2.6 \times 10^{-6} \mathrm{~cm}^{2} \mathrm{~V}^{-1} \mathrm{~s}^{-1}, d=180 \mathrm{~nm}$, and $T_{c}=370 \mathrm{~K}$, which corresponds to $E_{t} \sim 32 \mathrm{meV}$.

The switching of hole transport to single conduction mechanism, i.e., the trap model conduction in CdSe quantum dot dispersed P3HT thin films can be understood in terms of change in the hole transport parameters. In comparison of device 2 with device 1 parameters it is clearly seen that there is a reduction in the value of $E_{t}$ from 60 to $32 \mathrm{meV}$ and in $H_{b}$ from $2.5 \times 10^{18}$ to $1.7 \times 10^{18} \mathrm{~cm}^{-3}$ on incorporation of CdSe quantum dots in the host P3HT matrix. The decrease in the value $E_{t}$ implies that most of the traps are distributed close to HOMO thereby, even at low temperatures the charge carriers in the traps get sufficient energy to become detrapped and go into the extended states rather than remaining trapped in localized states as in case of pure P3HT, resulting in larger number of free charge carriers available for transport and hence the trap model conduction dominating at all temperatures. Further the enhancement in current density at all temperatures (the inset in Fig. 4 shows a specific case at $288 \mathrm{~K}$ for both devices 1 and 2) on dispersion of CdSe quantum dots is also being attributed to the above suggested mechanism based on the decrease in the values of $E_{t}$ and $H_{b}$. The reduction in hole mobility in device 2 , however, could be explained in terms of various well known ${ }^{15}$ forces that come into play for the stability of the CdSe quantum dots against coagulation in the P3HT composite. During the composite film formation when the polymer masks the CdSe quantum dots, the attractive van der waals forces acting between the quantum dots get dominated by the polymer induced steric repulsive forces ${ }^{15}$ and provide steric stability to the system. These resultant positive repulsive forces are experienced by holes, influencing their movement and expectedly allow them to scatter from their path hence the reduction in their mobility.

In conclusion it is demonstrated here that $J-V$ behavior in P3HT films on dispersion with CdSe quantum dots results in overall increase in hole current and switches the transport from dual conduction mechanism, viz., trap and mobility models to a only trap model. This has been attributed to a decrease in $E_{t}$ from 60 to $32 \mathrm{meV}$ and $H_{b}$ from 2.5 $\times 10^{18}$ to $1.7 \times 10^{18} \mathrm{~cm}^{-3}$.

${ }^{1}$ K. Kim, J. Liu, M. A. G. Namboothiry, and D. L. Carroll, Appl. Phys. Lett. 90, 163511 (2007)

${ }^{2}$ W. J. E. Beek, M. M. Wienk, and R. A. J. Janssen, Adv. Mater. (Weinheim, Ger.) 16, 1009 (2004).

${ }^{3}$ T. Shiga, K. Takechi, and T. Motohiro, Sol. Energy Mater. Sol. Cells 85, 1849 (2006).

${ }^{4}$ C. Y. Kwong, W. C. H. Choy, A. B. Djurisic, P. C. Chui, K. W. Cheng, and W. K. Chan, Nanotechnology 15, 1156 (2004).

${ }^{5}$ Y. Zhou, Y. Li, H. Zhong, J. Hou, Y. Ding, C. Yang, and Y. Li, Nanotechnology 17, 4041 (2006).

${ }^{6}$ W. U. Huynh, J. J. Dittmer, and A. P. Alivisatos, Science 295, 2425 (2002).

${ }^{7}$ N. C. Greenham, X. Peng, and A. P. Alivisatos, Phys. Rev. B 54, 17628 (1996).

${ }^{8}$ W. U. Huynh, J. J. Dittmer, W. C. Libby, G. L. Whiting, and A. P. Alivisatos, Adv. Funct. Mater. 13, 73 (2003).

${ }^{9}$ L. Qu, W. W. Yu, and X. Peng, Nano Lett. 4, 465 (2004).

${ }^{10}$ A. K. Kapoor, S. C. Jain, J. Pootmans, V. Kumar, and R. Mertens, J. Appl. Phys. 92, 3835 (2002).

${ }^{11}$ N. F. Mott and R. W. Gurney, Electronic Processes in Ionic Crystals (Oxford University Press, London, 1940).

${ }^{12}$ A. R. Brown, D. D. C. Bradley, J. H. Burroughes, R. H. Friend, N. C. Greenham, P. L. Burn, A. B. Holmes, and A. Kraft, Appl. Phys. Lett. 61, 2793 (1992).

${ }^{13}$ P. W. M. Blom, M. J. M. de Jong, and M. G. van Munster, Phys. Rev. B 55, R656 (1997).

${ }^{14}$ K. C. Kao and W. Hwang, Electrical Transport in Solids (Pergamon, Oxford, 1981), p. 160.

${ }^{15}$ P. C. Hiemenz and R. Rajagopalan, Principles of Colloid and Surface Chemistry (Marcel Dekker, New York, 1997), p. 575. 